\title{
ANTITUMOR TRANS PLATINUM ADDUCTS OF GMP AND AMP
}

\author{
Yangzhong Liu ${ }^{1}$, Maria F. Sivo ${ }^{2}$, Giovanni Natile ${ }^{2}$ and Einar Sletten*1 \\ ' Department of Chemistry, University of Bergen, Allegt.41, N-5007 Bergen, Norway \\ $<$ einar.sletten@kj.uib.no> \\ ${ }^{2}$ Department of Pharmaceutical Chemistry, University of Bari, Via E. Orabona 4, I-70125, Bari Italy
}

\begin{abstract}
Recently it has been shown that several analogues of the clinically ineffective trans-DDP exhibit antitumor activity comparable to that of cis-DDP. The present paper describes the binding of antitumor trans$\left[\mathrm{PtCl}_{2}(E \text {-iminoether })_{2}\right]$ (trans-EE) to guanosinemonophosphate (GMP) and adenosinemonophosphate (AMP). We have used HPLC and ${ }^{1} \mathrm{H}$ and ${ }^{15} \mathrm{~N}$ NMR to characterize the different adducts. In the case of a 1:1 mixture of trans-EE and GMP, at an early stage of the reaction, a monofunctional adduct is formed which, subsequently, is partly converted into a monosolvated monofunctional species. After about 70 hours an equilibrium is established between chloro and solvato monofunctional adducts at a ratio of 30/70. In the presence of excess GMP $(4: 1)$ the initially formed monofunctional adducts react further to give two bifunctional adducts, one with the iminoether ligands in their original $E$ configurations and the other with the iminoether ligands having one $E$ and the other, $Z$ configurations. The coordination geometry obtained by energy minimization calculations is in qualitative agreement with $2 D$ NMR data.
\end{abstract}

\section{INTRODUCTION}

The antitumor activity of cis-diamminedichloroplatinum(II) (cis-DDP), the most widely used metalbased anticancer drug, has been associated with formation of adducts with DNA (for recent reviews see [1]). Since the trans isomer of $c i s$-DDP is clinically ineffective it was assumed that a structure-activity relationship exists where only the cis geometry is therapeutically active. However, recently, it has been shown that several analogues of trans-DDP exhibit antitumor activity comparable to that of $c i s$-DDP [2-5]. Of particular interest is a group of iminoether derivatives analogous to trans-DDP: e.g. trans-[ $\left.\mathrm{PtCl}_{2}(\text { iminoether })_{2}\right]$ which was shown to be endowed with significant in vivo antitumor activity $[3,6]$. Surprisingly, these compounds were remarkably more cytotoxic than their congener $\operatorname{cis}$-[ $\mathrm{PtCl}_{2}$ (iminoether) $\left.)_{2}\right]$ [2].

In addition to the $c i$ and trans geometry, platinum-iminoether complexes can have either $E$ or $Z$ configuration depending on the relative position of the alkoxy group and the N-bonded $\mathrm{Pt}$ with respect to the $\mathrm{C}=\mathrm{N}$ double bond, and this represents another aspect to be considered for the evaluation of structure-activity relationships. The DNA binding mode of trans-EE in a cell free medium has been characterized by several biophysical methods [7]. As a result of these studies it has been concluded that kinetically stable trans-EE forms monofunctional adducts at guanine residues in double-helical DNA. As a consequence trans-EE is assumed to modify DNA differently to clinically ineffective trans-DDP.

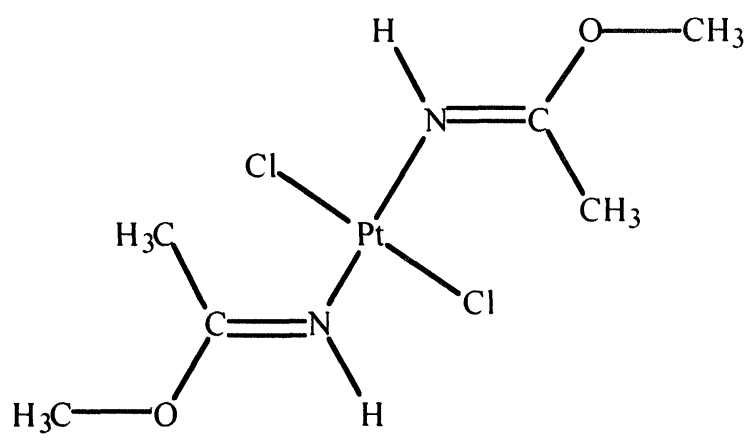

Trans- $\left[\mathrm{PtCl}_{2}\{E-\mathrm{HN}=\mathrm{C}(\mathrm{OMe}) \mathrm{Me}\}_{2}\right]($ trans $-E E)$

The nature of the non-leaving groups, i.e. the amine ligands in trans-DDP and the iminoether ligands in trans-EE, somehow play an important role and it has been speculated that in the latter case one of the drug - DNA interactions could not involve directly the metal ion but be mediated by the iminoether ligand. Such a type of interaction with DNA would place the platinum-iminoether complex at the junction between'c'is-DDP and non-metallic cytotoxic agents. 
Recently, a detailed HPLC and NMR spectroscopic analysis of the DNA duplex consisting of trans$E E$ platinated $5^{\prime}-\mathrm{d}\left(C_{C C T C G}{ }^{*}\right.$ CTCTC) $\left(\mathrm{I}_{\mathrm{M}}\right)$ and its complement $5^{\prime}$-d(GAGAGCGAGG) (II) was described [8]. The analysis revealed that the binding pattern of trans-EE is more complex than first anticipated involving $\mathrm{pH}$ and sequence-dependent isomerization reactions and deplatination when the NMR sample contains high $\mathrm{NaCl}$ concentration $(0.2 \mathrm{M})$. This paper describes a more detailed picture of the binding pattern between trans-EE and mononucleotides (GMP and AMP).
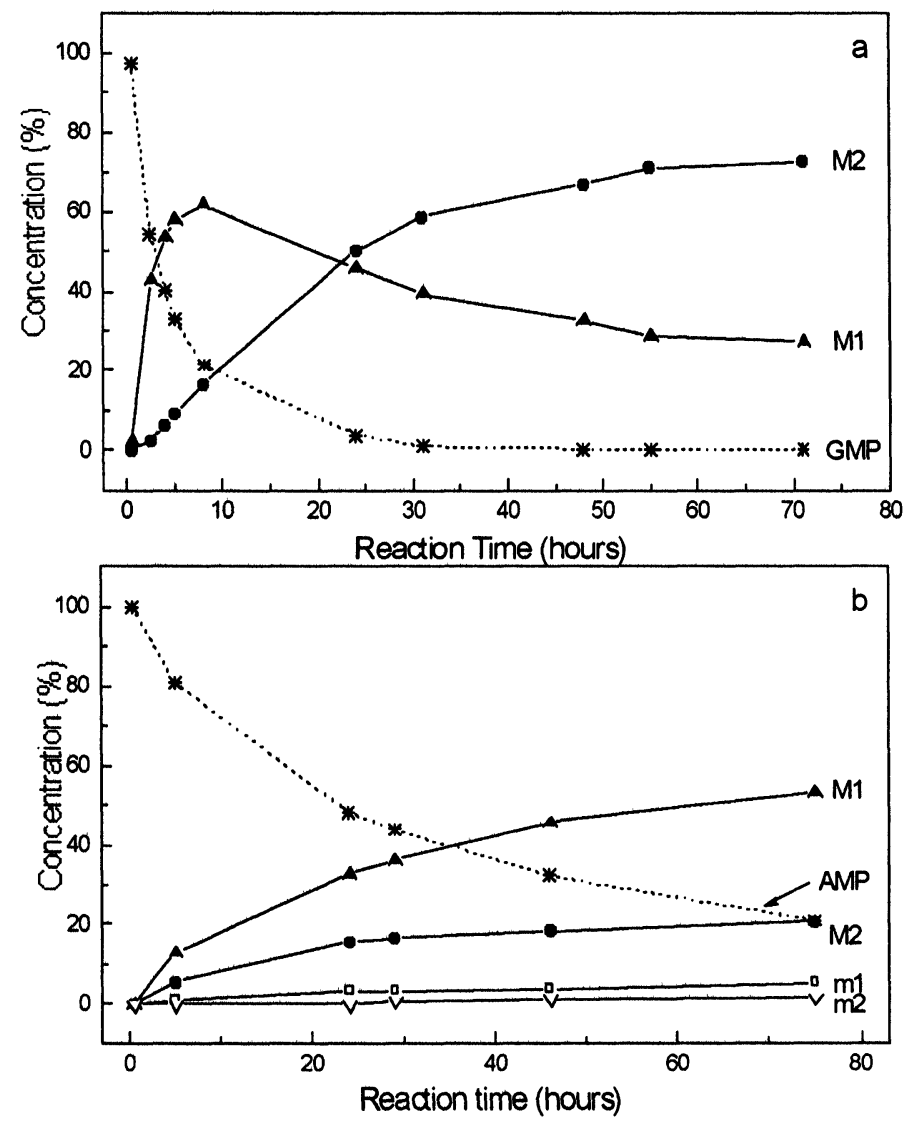

Figure 1. Distribution curves for the trans-EE platination reaction of nucleotides at 1:1 molar ratio.

(a) GMP (*), (b) AMP. at pH 6.8 in $\mathrm{H}_{2} \mathrm{O}, 298 \mathrm{~K}$.

\section{MATERIALS AND METHODS \\ Sample preparation.}

The trans-EE complex was synthesized by the Bari group as previously described [9]. 5'riboguanosinemonophosphate (sodium salt) (GMP) and the corresponding adenine phosphate (AMP) were purchased from Sigma and used without further purification. Deuterium oxide was purchased from Flurochem Limited Company. The reactions between trans-EE and the mononucleotides were performed using the $\mathrm{PtCl}_{2}[E-\mathrm{HN}=\mathrm{C}(\mathrm{OMe}) \mathrm{Me}]_{2}$ and the nucleotide in molar ratios 1:1 and 4:1, respectively, at $\mathrm{pH}$ 6.8. The solutions were kept in the dark at room temperature. The platinated nucleotides were purified by HPLC on a Waters 626 LC instrument using the Millenium 32 software [10]. A reverse phase Waters Symmetry C8 column was used with gradient eluent $(0-50 \%$ methanol) in $50 \mathrm{mM} \mathrm{NaClO}$ at a flow rate of $0.8 \mathrm{ml} / \mathrm{min}$. The reactions were monitored by HPLC until the equilibrium was reached. The platinated samples for NMR experiments were separated by HPLC, lyophilized and dissolved into $0.5 \mathrm{ml} \mathrm{D}_{2} \mathrm{O}$. No buffer was added and the $\mathrm{pH}$ value of the solution was about 4 . The samples of unplatinated GMP and AMP were dissolved in 0.5 $\mathrm{ml} \mathrm{D}_{2} \mathrm{O}, \mathrm{pH} 5.5$ (final concentration: $0.1 \mathrm{M}$ ).

NMR Spectroscopy.

NMR experiments were performed on a Bruker DRX 600 instrument, operating at $600 \mathrm{MHz}$ for ${ }^{1} \mathrm{H}$ NMR spectroscopy. 1 ${ }^{1} \mathrm{H}$ NMR spectra were collected with a total of $32 \mathrm{~K}$ complex points and 64 transients. For all 1D and 2D spectra the spectral width were $7200 \mathrm{~Hz}$ and a relaxation delay of $2 \mathrm{~s}$ was used. The presaturation pulse sequence was used for the samples in $\mathrm{D}_{2} \mathrm{O}$ and the 3-9-19 WATERGATE pulse sequence was used for the samples in $\mathrm{H}_{2} \mathrm{O}$ solution for water suppression [11]. In the ROESY and NOESY experiments, a total of 2048 complex points in $t_{2}$ were collected for each of $256 t_{1}$ increments and 80 
transients were averaged for each increment. Two-dimensional $\left[{ }^{1} \mathrm{H},{ }^{15} \mathrm{~N}\right] \mathrm{HMQC}$ spectra with $1 \mathrm{~K}$ complex points along $\mathrm{t}_{2}$ were recorded for 128 transients. The experiment was optimized for two-bond ${ }^{1} \mathrm{H}-{ }^{15} \mathrm{~N}$ connectivity involving nonexchangeable protons, where $\Delta=1 / 2 J_{\mathrm{NH}}=48 \mathrm{~ms}$. Coupling constants are found in the range of 6-14 Hz, except for ${ }^{2} J_{\mathrm{N} 9-\mathrm{Hl}}$, which is found to be $4.1 \mathrm{~Hz}$ in GMP. The ${ }^{1} \mathrm{H}^{9}{ }^{9} \mathrm{~N}$ spectra widths were 1929 and $6082 \mathrm{~Hz}$ for free GMP and 1912 and $12064 \mathrm{~Hz}$ for the trans-EE adduct, respectively. The FID's were multiplied by a square-cosine function and zero-filled to 2048 complex points along $t_{1}$ and $t_{2}$. The NMR data were processed on a Silicon Graphics INDY workstation using the program FELIX (Biosym) [12] and on a $133 \mathrm{MHz}$ Pentium PC using 1D and 2D WIN-NMR (Bruker) [13]. The one dimensional 'H FID's were multiplied by an exponential window function prior to Fourier transformation. Typically, $0.3 \mathrm{~Hz}$ was added to the line widths. No baseline correction was needed. ${ }^{1} \mathrm{H}$ spectra were referenced to the HDO-resonance at $4.76 \mathrm{ppm}$ at $298 \mathrm{~K}$ and ${ }^{15} \mathrm{~N}$ spectra relative to saturated $\mathrm{NH}_{4} \mathrm{Cl}$ solution at $-352.9 \mathrm{ppm}$.

\section{RESULTS}

\section{HPLC analysis}

Small fractions of the 1:1 trans-EE/GMP reaction mixture were analyzed by HPLC at several time intervals. The species distribution is given in Figure 1a. Initially, two species M1 and M2 are formed at different rates, the faster forming species, $\mathrm{Ml}$, attains a maximum concentration after ca 10 hours and is then partly converted to a second species, $\mathrm{M} 2$; at equilibrium the ratio $\mathrm{M} 1 / \mathrm{M} 2$ is $c a .30: 70$. The reaction proceeds with a half-time of $3.5 \mathrm{~h}\left(\right.$ at $\left.298 \mathrm{~K}, \mathrm{c}_{\mathrm{Pt}_{\mathrm{t}}}=1 \mathrm{mM}\right)$.

For the reaction with excess GMP $(4: 1)$ a more complex mixture of products was formed. Similarly to the case of a 1:1 ratio of reactants, two monofunctional adducts M1 and M2 are formed initially and are then converted into two bifunctional species (M3 and M4) which reach a concentration ratio of 5:1 after 20 days and have chromatographic retention times of 34 and $33 \mathrm{~min}$., respectively. For longer reaction time the amount of M4 increased further and after two months exceeded that of M3.

For AMP the reaction is much slower than for GMP (Figure $1 \mathrm{~b}$ ). The half-time $\mathrm{t}_{1 / 2}=23 \mathrm{~h}$ is estimated from the HPLC trace. After about 7 days about $91 \%$ of AMP has reacted. As for GMP two major species M1 $60 \%)$ and M2 (20\%) are formed, a chloro and an aquacomplex, respectively. In addition, two minor species (less than $5 \%$ ) are formed, probably representing the corresponding chloro and aqua N1 platinated complexes.

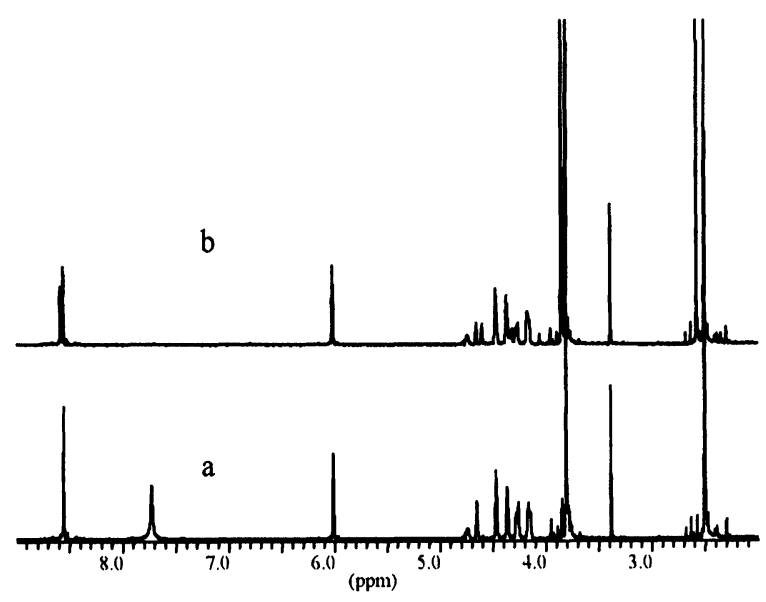

Figure 2. 'H NMR spectra for adduct $\mathrm{Ml}$ recorded at different time intervals, $\mathrm{pH} 4.8$, in $\mathrm{D}_{2} \mathrm{O}$ at $298 \mathrm{~K}$.

(a) newly prepared sample. (b) 20 hours later.

(b) (The peak at $3.4 \mathrm{ppm}$ is the trace residue of methanol from HPLC eluent.)

\section{NMR assignments}

Monofunctional GMP adducts. The 1D 'H NMR spectra were recorded at different time intervals for the reaction mixture and for the separate HPLC fractions. Figure 2 exhibits the $1 D$ spectra for MI recorded at different time intervals. Integration of the 1D spectra confirms the 1:1 ratio of GMP to trans-EE, (1:6 of H8 to $\mathrm{CH}_{3}$ or $\mathrm{CH}_{3} \mathrm{O}$ peak). The $\mathrm{NH}$ proton of the iminoether ligands exchanges slowly with $\mathrm{D}_{2} \mathrm{O}$ during $2 \mathrm{D}$ experiment at $298 \mathrm{~K}$ and disappear after about 20 hours. The proton chemical shifts of the resonance of the different platinated species are listed in Table 1.

The G-H8 resonance for $\mathrm{M} 1$ and $\mathrm{M} 2$ are shifted $0.46 \mathrm{ppm}$ and $0.40 \mathrm{ppm}$ downfield relative to unplatinated GMP, respectively. Platinum coordination at N7 is usually found to produce $\mathrm{H} 8$ downfield shifts in the $0.5-1 \mathrm{ppm}$ range as a result of electron-withdrawing effect of the metal. The resonances of the sugar 
protons exhibit only insignificant chemical shift changes relative to native GMP. Also, the proton chemical shifts of the methyl and the methoxide groups, $2.46 \mathrm{ppm}$ and $3.78 \mathrm{ppm}$, respectively, are almost identical to those of free trans-EE in solution [9].

The two species, $\mathrm{M} 1$ and $\mathrm{M} 2$, interchange reversibly depending on $\mathrm{pH}$ and chloride concentration. The equilibrium ratio $\mathrm{M} 1 / \mathrm{M} 2=0.35$ at $\mathrm{pH} 6.8$ increases to 0.7 at $\mathrm{pH} 1.7$. Addition of $\mathrm{NaCl}$ to the sample also induces a conversion of $\mathrm{M} 2$ to $\mathrm{M} 1$ indicating that the latter is the chloro complex and $\mathrm{M} 2$ the aqua complex. Plots of G-H8 chemical shifts $v s$. $\mathrm{pH}$ for M1 and M2 are shown in Figure 3. The $\mathrm{pK}_{\mathrm{a}}$ at about 8.5 corresponds to guanine N1 deprotonation. The absence of change in chemical shift at $\mathrm{pH}<3$ is clearly diagnostic for GN7 platination. The change of chemical shift at 5.8 corresponds to the influence of phosphate (de)protonation.

Table 1. ${ }^{1} \mathrm{H}$ NMR chemical shifts (ppm) for GMP and trans- $E E$ platinated adducts. Reference: HDO signal at $4.76 \mathrm{ppm}, 298 \mathrm{~K}, \mathrm{pH}$ in parentheses.

\begin{tabular}{|l|l|l|l|l|l|}
\hline $\begin{array}{l}\delta \\
(\mathrm{ppm})\end{array}$ & GMP (7.1) & M1 (4.8) & M1 (6.8) & M2 (4.8) & M2 (6.8) \\
\hline $\mathrm{H} 8$ & 8.17 & 8.55 & 8.63 & 8.58 & 8.57 \\
\hline $\mathrm{H} 1^{\prime}$ & 5.91 & 6.01 & 5.96 & 6.02 & 5.94 \\
\hline $\mathrm{H} 2^{\prime}$ & $--^{-a}$ & 4.65 & 5.96 & 4.60 & 5.94 \\
\hline $\mathrm{H} 3^{\prime}$ & 4.46 & 4.47 & 4.46 & 4.46 & 4.45 \\
\hline $\mathrm{H}^{\prime}$ & 4.30 & 4.36 & 4.34 & 4.37 & 4.33 \\
\hline $\mathrm{H}^{\prime}$ & 3.98 & 4.27 & 4.07 & 4.32 & 4.05 \\
\hline $\mathrm{H}^{\prime \prime}$ & 3.98 & 4.15 & 4.09 & 4.15 & 4.03 \\
\hline $\mathrm{NH}^{\mathrm{b}}$ & - & 7.73 & 7.78 & 7.92 & 7.95 \\
\hline $\mathrm{OCH}_{3}$ & - & 3.81 & 3.76 & 3.85 & 3.78 \\
\hline $\mathrm{CH}_{3}$ & - & 2.50 & 2.45 & 2.58 & 2.46 \\
\hline
\end{tabular}

a. Overlap with residual $\mathrm{HDO}$ at $4.7-4.8 \mathrm{ppm}$

b. $\mathrm{NH}$ protons are slowly exchanged in $\mathrm{D}_{2} \mathrm{O}$ during $10-20$ hours.

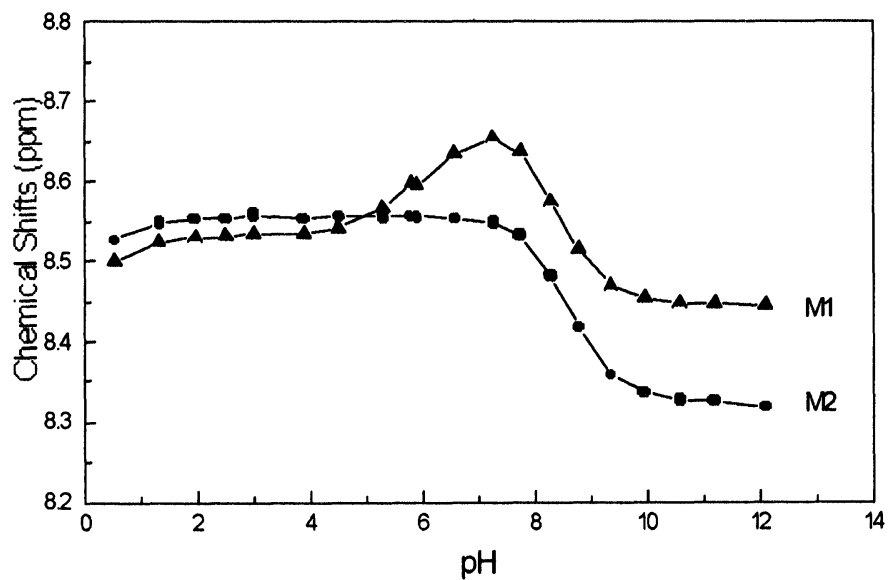

Figure 3. Titration curves for $\mathrm{G}-\mathrm{H} 8$ of adducts $\mathrm{Ml}$ and $\mathrm{M} 2$. $\left(298 \mathrm{~K}\right.$ in $90 \% \mathrm{H}_{2} \mathrm{O}$ )

The 2D ${ }^{1} \mathrm{H}$ ROESY spectra for both M1 and M2 show a medium size NOE crosspeak between G-H8 and $\mathrm{CH}_{3}$ of trans-EE. A large cross peak between $\mathrm{H} 8$ and $\mathrm{CMe}$ is expected since both are cis to platinum with respect to the $\mathrm{C}=\mathrm{N}$ double bound and therefore can come very close to each other during rotation of the ligands about the $\mathrm{Pt}-\mathrm{N}$ bonds. No crosspeak corresponding to $\mathrm{G}-\mathrm{H} 8 \ldots \mathrm{CH}_{3} \mathrm{O}$ was detected above the noise level. A strong intra ligand crosspeak is observed between the imino proton and the methoxide group confirming that the iminoether ligands retain the configuration also after coordination of GMP.

Bi-functional adducts. The bifunctional adducts M3 and M4 were characterised by ${ }^{1} \mathrm{H}$ and ${ }^{15} \mathrm{~N}$ NMR spectroscopy. The 2:1 ratio of GMP to trans-EE ratio was confirmed by integration of $\mathrm{H} 8$ and $\mathrm{CH}_{3}(1: 3$ peak intensity). The G-H8 proton chemical shifts for the two isomers are almost identical to those of the M1 and M2 adducts. The main difference between the spectra of M3 and M4 is that the methyl and methoxide groups give rise to one set of signals for M3 but two sets for M4 (Figure $4 \mathrm{a}, \mathrm{b}$ ). 

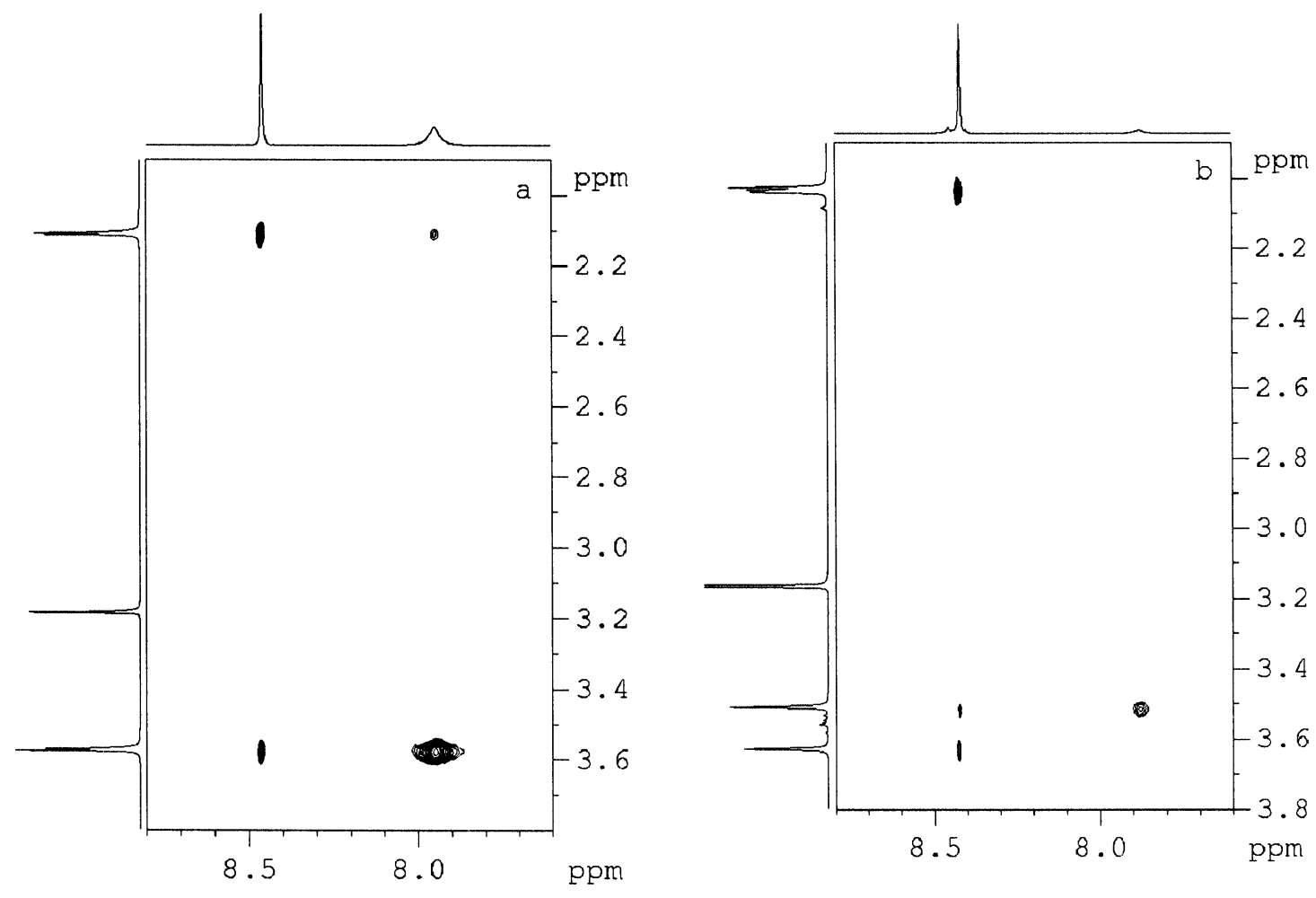

Figure 4. Selected regions of the ROESY spectrum of the bifunctional GMP/trans-EE adducts showing contacts between G-H8 and the methyl and methoxide groups. The intra $E$-iminoether ligand cross peaks involving $\mathrm{OMe}$ and $\mathrm{N}-\mathrm{H}$ are also apparent. (a) M3, (b) M4

The ROESY spectrum of M3 shows two distinct crosspeaks involving H8, one strong to the methyl group and one less intense to the methoxide group. In addition a strong intra-ligand crosspeak is observed between the methoxide group and the imino proton. In M4 the two partly overlapping methyl signals are both coupled to $\mathrm{H} 8$ while the crosspeak between $\mathrm{OMe}$ and $\mathrm{NH}$ is much greater for the isomerized iminoether $(\mathrm{Z}$ configuration) than for the non isomerized one (E configuration)

${ }^{15} \mathrm{~N}$ NMR is a sensitive probe for detecting specific nitrogen binding sites on nucleobases. The use of ${ }^{1} \mathrm{H}$-detected ${ }^{1} \mathrm{H}-{ }^{15} \mathrm{~N}$ HMQC spectroscopy greatly enhances the sensitivity of natural abundance ${ }^{15} \mathrm{~N}$ spectroscopy. The ${ }^{15} \mathrm{~N}$ resonances of $\mathrm{N} 7$ and $\mathrm{N} 9$ are easily observed through multi-bond scalar coupling with G-H8. A cross peak between N9 and the anomeric proton $\mathrm{H}^{\prime}$ confirms the assignments. For the transEE/GMP M3 complex the N7 signal is shifted to $-238.4 \mathrm{ppm}(97,5 \mathrm{ppm}$ upfield that of free GMP), while N9 exhibits a minor shift to $-202.9 \mathrm{ppm}(4.3 \mathrm{ppm}$ downfield that of free GMP). The large upfield shift induced by platination is comparable to similar shifts observed for protonation of purine nitrogens ( $\sim 70 \mathrm{ppm})$.

The AMP adduct. The trans-EE binding to AMP follows the same pattern as that observed_for GMP. A-H8 is shifted $0.4 \mathrm{ppm}$ downfield while A-H2 is not affected. ${ }^{1} \mathrm{H}-{ }^{15} \mathrm{~N}$ HMQC spectroscopy of platinated AMP (M1) shows significant upfield shift of N7 (-247.7 ppm as compared to a free AMP N7 value of $-150.8 \mathrm{ppm})$ while $\mathrm{N} 3$ and $\mathrm{N} 9$ are only shifted downfield by about $10 \mathrm{ppm}$. This shift confirms the N7 binding of AMP in trans$E E$ adducts. of $\mathrm{Ml}$ at $\mathrm{pH} \mathrm{4,} \mathrm{N} 1$ is partly protonated, therefore, the $\mathrm{N} 1$ signal shifts to high field by $22.3 \mathrm{ppm}$. ROESY spectra show the AMP adduct to have a relationship to the iminoether ligand similar to GMP. A medium sized ROESY crosspeak is observed for the A-H8 $\mathrm{CH}_{3}$ contact. Another very weak crosspeak is observed for the A- $\mathrm{H} 8 \mathrm{CH}_{3} \mathrm{O}$ contact. In addition, the anomeric proton has weak contacts to the methyl group.

Molecular modeling. In the molecular modeling approach using the BIOSYM software [14] for AMBERminimized energies the trans- $E E$ complex was fixed in the geometry adopted in the solid state [9] except for the methyl groups which were replaced by pseudoatoms calculated from averaging the hydrogen X-ray coordinates. The two iminoether ligands are not coplanar in the X-ray structure but are twisted $35^{\circ}$ to relieve steric crowding of the methyl groups. In the calculation the configuration of the iminoether ligands were fixed but allowed to rotate freely about the Pt-NH bonds. Also the GMP ligand was allowed to rotate freely around the Pt-N7 bond while keeping the square-planar geometry fixed. Both the monofunctional and the bifunctional models were subjected to energy minimization. 

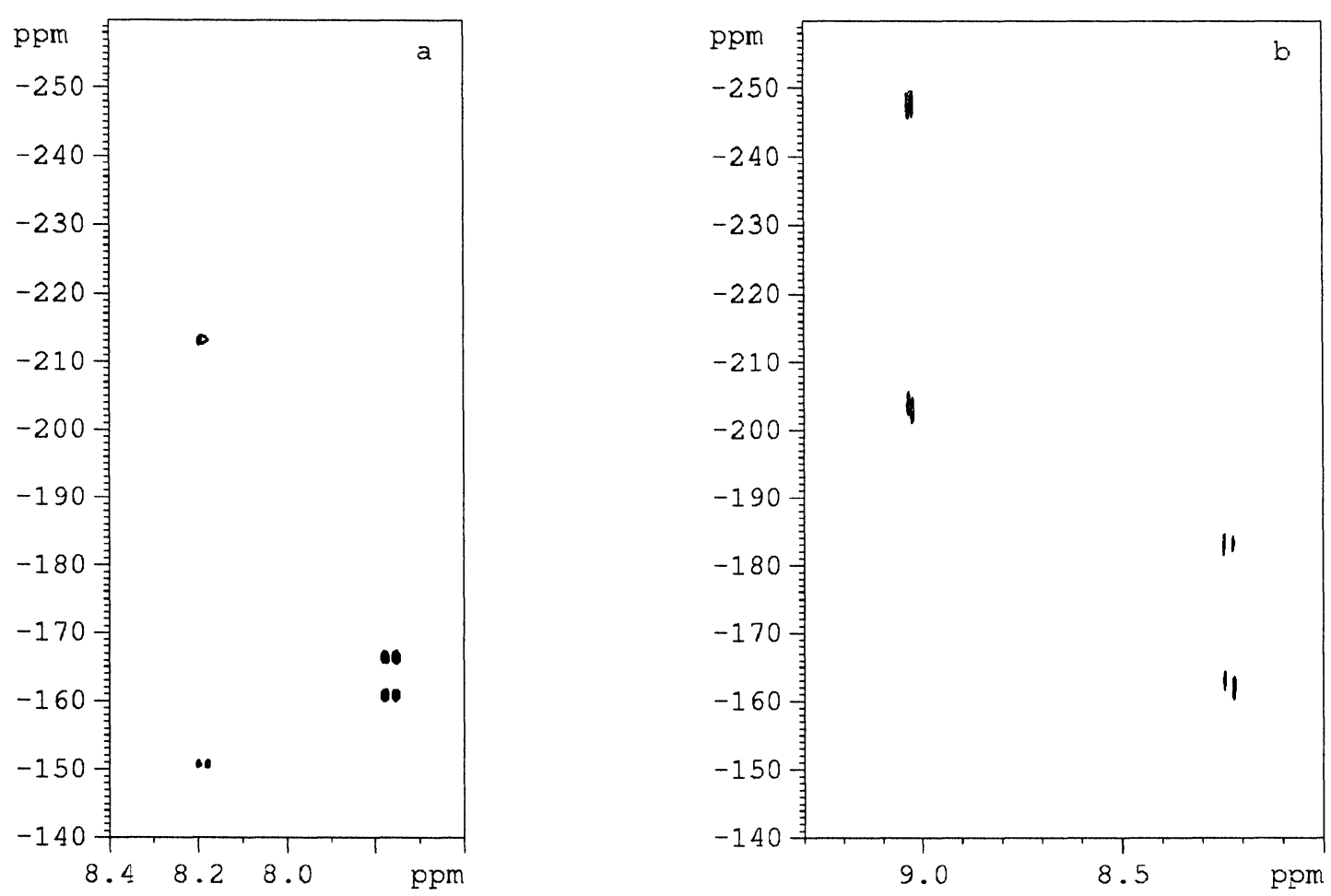

Figure 5. ${ }^{1} \mathrm{H}-{ }^{15} \mathrm{~N}$ correlation NMR spectrum at $280 \mathrm{~K}$. (a) AMP, pH 7, (b) trans-EE adduct M1, pH 4.

No symmetry constraints were applied to the relative positions of the chemically equivalent GMP ligands. The computational details are given in ref. [8]. One may add that most probably there exixts a distribution of several conformers. However, the NMR spectra were recorded at a temperature at which the signals from different conformers are not resolved. A stereo-view of the refined molecular model is shown in Figure 6. Inter and intra through-space $\mathrm{H}-\mathrm{H}$ contacts are in qualitative agreement with the NMR data. The $\mathrm{H} 8 \cdots \mathrm{CH}_{3}$ distance of $3.47-3.70 \AA$ is in agreement with a relatively strong cross peak while the $\mathrm{H} 8 \cdots \mathrm{CH}_{3} \mathrm{O}$ distance of 4.49-4.93 Å gives a comparatively weak cross peak.

\section{DISCUSSION}

Previously we have reported on the NMR structure of an oligonucleotide duplex platinated by trans$E E$ at a guanine $\mathrm{N} 7$ site [8]. Only the major monofunctional adduct was structurally characterized, however, two minor species were also present as evidenced by HPLC analysis. In this context it is of interest to explore the relation between monofunctional $v s$ bifunctional adduct formation which is closely related to the ability to engage in inter and intra strand cross-linking reactions. Other trans-Pt complexes with bulky ligands, e.g. pyridine, have been reported to have considerable enhanced ability to form interstrand cross-links over both its cis isomer and the $\mathrm{NH}_{3}$ complexes [15]. This finding is contrary to the situation for DNA incubated with trans-EE for $48 \mathrm{hr}$ at $37^{\circ} \mathrm{C}$ in $10 \mathrm{mM} \mathrm{NaClO}{ }_{4}$ which was shown to produce only marginal amounts of interstrand cross links [16]. Bierback and Farell [17] also showed that this reaction is drastically slowed $\left(\mathrm{t}_{1 / 2}=\right.$ $17 \mathrm{~h}, \mathrm{~T}=310 \mathrm{~K}$ ) when one of the ammine ligands was replaced by quinoline. This latter compound exhibits the same tendency as trans-EE to form long-lived monofunctional adducts on DNA, clearly different from the behavior of trans-DDP. 

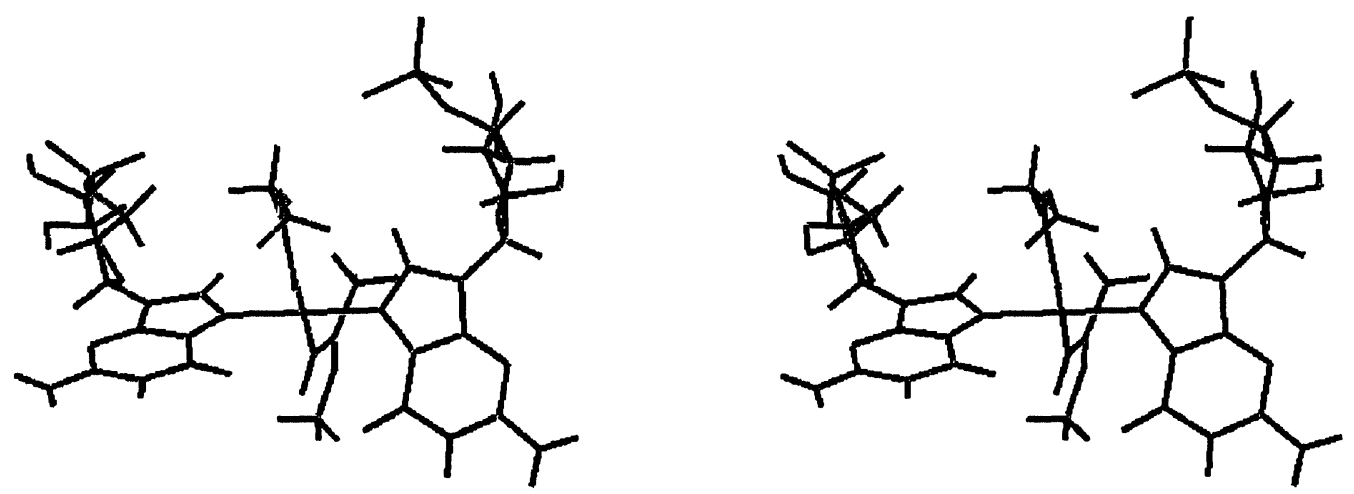

Figure 6. Stereoview of the energy minimized molecular model of the bifunctional GMP/trans-EE adducts.

Comparison of reaction rates for trans-EE platination of mononucleotides and oligonucleotides indicates that the trans-EE platination of the G-residue in the single strand oligonucleotide $\mathrm{d}$ (CCTCGTCTC) proceeds at about the same rate as for the mononucleotide (Figure 7) [8]. For double helical DNA the geometric restraints may drastically reduce the trans- $E E$ platination rate.

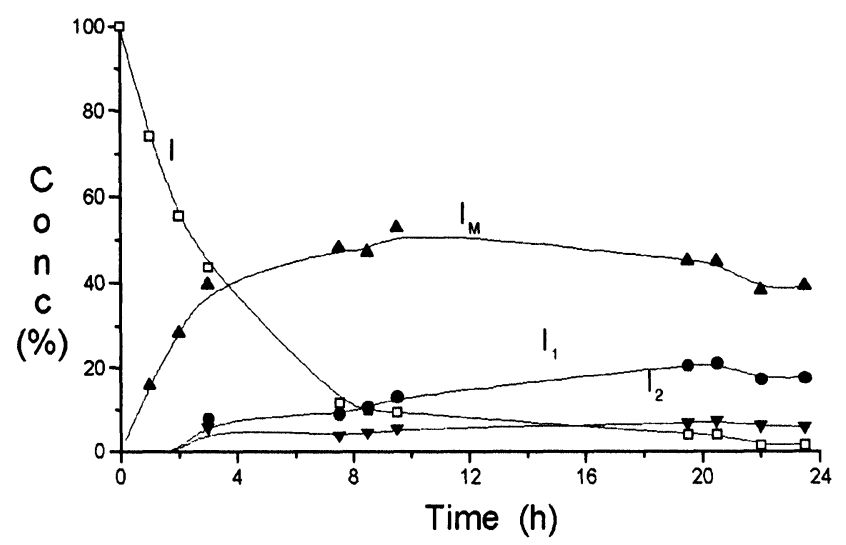

Figure 7. Species distribution curves for trans- $E E$ platination of $\mathrm{d}(\mathrm{CCTCGCTCTC})$ :

(A) Major adduct $\left(\mathrm{I}_{\mathrm{M}}\right),(\mathbf{O})$ and $(\boldsymbol{\nabla})$ Minor adducts, $(\mathrm{I}$ non-platinated oligonucleotide.

$\mathrm{pH} 2.5$, no salt, at room temperature in $\mathrm{H}_{2} \mathrm{O}$. (From ref. [8]).

The difference between the GMP adducts M1 and M2 is deduced from monitoring the equilibrium as a function of $\mathrm{pH}$ and addition of $\mathrm{NaCl}$. Most likely $\mathrm{M} 1$ represents the GMP chloro complex and $\mathrm{M} 2$ the corresponding solvato species. From the ROESY spectra it is evident that, in both M1 and M2, there are strong crosspeaks between H8 of GMP and the C-Me groups of the iminoether ligands which come very close during rotation of the ligands about the Pt-N bonds.

With excess GMP $(4: 1)$ the initially formed monofunctional adducts are converted to bifunctional trans-EE(GMP) 2 adducts (M3 and $\mathrm{M} 4)$. The transition is quite slow and takes approximately 15 days to be completed at $298 \mathrm{~K}$ and $\mathrm{pH} 6.8$. The retention time for M3 and M4 are surprisingly close to the retention time for the monofunctional adducts. The M3 bifunctional adduct has preserved the configuration of the iminoether ligand $(E)$ while in the M4 bifunctional adduct one iminoether ligand has isomerized to the Z configuration. The concentration of the M4 isomer is initially only $1 / 5$ of M3, however, after two months the ratio has increased to approximately $1: 1$. This implies that M4 has similar stability to that of M3. This is a rather unexpected result since in the starting dichloro species the isomer with both iminoether ligands in the $E$ configuration is far more stable than any other isomer. Therefore it appears that the ancillary ligands (the purine bases in this case) are able to influence the thermodynamic stability of the iminoether configurations. Similar geometry conversion processes were found in nucleoside-trans-EE adducts (unpublished results).

ACKNOWLEDGMENTS. Financial support by the European Commission BIOMEDII program (Contract BMH4-CT97-2485) and COST action D8/007/97 and D8/012/97 are gratefully acknowledged. Thanks are extended to the Norwegian Research Council for financial support (Contract 135055/410) and the Italian M.U.R.S.T.(Cofin $1988 \mathrm{n}^{\circ} 9803021072$ ). 
1. B. Lippert (ed.) Cisplatin. Chemistry and Biochemistry of a Leading Anticancer Drug. Wiley-VCH, Zurich, 1999

2. G. Natile, M. Coluccia, in: Topics in Biological Inorganic Chemistry, Metallopharmaceuticals I, DNA Interactions. 1, 73-98, (1999) (M. J. Clarke, P. J. Sadler, eds). Springer-Verlag, Berlin.

3. M. Coluccia, A. Nassi, F. Loseto, A. Boccarelli, M.A. Mariggio, D. Giordano, F.P. Intini, P. Caputo, G. Natile. J. Med. Chem. (1993) 36, 510-512.

4. N. Farrell, in: Metal Ions in Biological Systems. A. Sigel and H. Sigel (eds.) (1996) 32, 603-639. Marcel Dekker, Inc., New York. N. Farrell, L.R. Kelland, J.D. Roberts, M. Van Beusichem. Cancer Res. (1992) 52, 5065-5072.

5. L.R. Kelland, C.F.J. Barnard, I.G. Evans, B.A. Murrer, B.R.C. Theobald, Wyer, S.B., P.M. Goddard, M. Jones, M. Valenti, A. Bryant, P.M. Rogers, K.R. Harrap. J. Med. Chem. (1995) 38, 3016-3024.

6. M. Coluccia, A. Boccarelli, M.A. Mariggio, N. Cardellicchio, P. Caputo, F.P. Intini, G. Natile. Chem. Biolog. Interactions, (1995), 98, 251-266.

7. R. Zaludova, G. Natile, V. Brabec. Anti-Cancer Drug Design (1997), 12, 295-309.

8. B. Andersen, N. Margiotta, M. Coluccia, G. Natile, E. Sletten. Metal-Based Drugs (2000) 7, 23-32.

9. R. Cini, P.A. Caputo, F.P. Intini, G. Natile. Inorg. Chem. (1995) 34, 1130-1137.

10. Waters Millenium 32 software.

11. V. Sklenar, M. Piotto, R. Leppik, V. Saudek. J. Magn. Reson. A (1993) 102, 241-245.

12. FELIX (version 2.30) Processing package, BIOSYM/MSI.

13. Bruker ID WIN-NMR (version 960901.2) and 2D WIN-NMR (version 6.02).

14. BIOSYM User Guide for Insight II (version 2.3.5), Discover (version 2.9.5).

15. Y. Zou, B. Van Houten, N. Farell. Biochemistry (1993) 32, 9632-9638.

16. V. Brabec, O. Vrana, O. Novakova, V. Kleinwachter, F.P. Intini, M. Coluccia, G. Natile. Nucleic Acids Res. (1996) 24, 336-341.

17. U. Bierbach and N. Farell. Inorg. Chem. (1997) 36, 3657-3665.

\section{Received: August 16, 2000- Accepted: September 11, 2000 -} Received in revised camera-ready format: September 14, 2000 